\title{
Le décret du 14 frimaire an II sur l'assèchement des étangs : folles espérances et piètres résultats. L'application du décret en Brie
}

Jean-Michel Derex

\section{(2) OpenEdition \\ Journals \\ Édition électronique \\ URL : https://journals.openedition.org/ahrf/449 \\ DOI : $10.4000 /$ ahrf.449 \\ ISSN : 1952-403X \\ Éditeur : \\ Armand Colin, Société des études robespierristes}

Édition imprimée

Date de publication : 1 septembre 2001

Pagination : 77-97

ISSN : 0003-4436

Référence électronique

Jean-Michel Derex, «Le décret du 14 frimaire an II sur l'assèchement des étangs : folles espérances et piètres résultats. L'application du décret en Brie ", Annales historiques de la Révolution française [En ligne], 325 | juillet-septembre 2001, mis en ligne le 10 avril 2006, consulté le 22 avril 2022. URL : http:// journals.openedition.org/ahrf/449; DOI : https://doi.org/10.4000/ahrf.449

Ce document a été généré automatiquement le 22 avril 2022

Tous droits réservés 


\title{
Le décret du 14 frimaire an II sur l'assèchement des étangs : folles espérances et piètres résultats. L'application du décret en Brie
}

\author{
Jean-Michel Derex
}

1 La législation concernant le monde de l'eau fut très réactive aux événements's . Le décret du 14 frimaire an II (4 décembre 1793) sur l'assèchement des étangs de la République en est un bon exemple. Cet article a pour but de faire le point sur l'application de cette législation montagnarde prise dans le domaine agricole et de mesurer l'écart qui a pu exister entre les ambitions des cercles politiques parisiens lors de l'élaboration du décret et les applications des textes sur le terrain'.

I-Les espoirs suscités par le vote du décret du 14 frimaire

2 Le vote du décret du 14 frimaire concernant l'assèchement des étangs fut précédé par trois rapports et projets: celui de Eschasseriaux' sur le dessèchement des marais (septembre 1792), celui de Rougier-Labergerie sur le dessèchement des étangs marécageux (11 septembre 1792), et un troisième de Boudin'sur le dessèchement et la mise en culture des étangs de la Sologne, de la Bresse et de la Brenne (octobre 1793)'. La simple lecture des titres des projets de lois permet de constater un glissement de l'objet traité (marais puis étangs marécageux et enfin étangs) ${ }^{*}$ et montre que le problème fut posé en termes de rupture par rapport à la période précédente'.

3 Ces rapporteurs présentent des points de vues proches les uns des autres. Eschasseriaux dénonçait l'inefficacité et l'injustice du système jusque-là en vigueur: «Ce qu'on a fait jusqu'ici paraît avoir été dirigé par un mauvais système. Jusqu'ici les entreprises de dessèchement ont été livrées aux particuliers, à des compagnies de dessècheurs ou à des concessionnaires. Le décret du 26 décembre 1790 est tantôt une invitation faible, tantôt une loi injuste ». Boudin quant à lui faisait de même $\left(^{(0)}\right.$ : «Le décret du 26 décembre 1790 sur lequel on avait fondé tant d'espérance n'a pas fait et ne 
peut pas faire dessécher un seul arpent ". Convergence de vues aussi avec les arguments concernant l'hygiène Eschasseriaux qualifiait les étangs de «cloaques mortifères, dont l'avidité féodale et la superstition avait couvert le sol et empoisonné de si belles contrées [...] Il faut se hâter de détruire la cause de cette influence de l'air sur la vie humaine. La législation veille à la liberté, à la sûreté, à la propriété des hommes quel plus grand bienfait peut-elle leur rendre que de s'occuper de purifier l'air qu'ils respirent et le sol sur lequel leur destinée les a placé ? »("). Convergence encore sur les buts politiques à poursuivre, l'assèchement des étangs s'inscrivant à la fois dans le cadre de la politique anti-cléricale et anti-seigneuriale du moment Boudin notait aussi que « la consommation du maigre diminuera par le relâchement de l'abstinence et le dégoût de la monasticité... À présent que les monastères sont supprimés sans retour et que l'observance du maigre s'affaiblira à mesure que la raison et le républicanisme feront des progrès. L'effet doit cesser avec la cause ». Ce décret s'en prenait aussi à l'un des symboles du système seigneurial l'étang. Jean Boutier a bien montré cette attaque de l'étang comme symbole du bien seigneurial. Il parle à ce propos d'une guerre des étangs au printemps et à l'été 1791 avec l'ouverture de bon nombre d'étangs du BasLimousin". Convergence de vue enfin sur la manière à employer pour assécher ces zones humides Boudin déclarait que "tout est réuni pour imposer à la Convention nationale l'obligation de substituer à la loi du 26 décembre 1790 un mode de dessèchement vraiment révolutionnaire et exécutable». Il demandait pour cela l'intervention du gouvernement «Il n'y a qu'un agent actif et puissant qui puisse opérer le dessèchement des marais de la République, c'est le gouvernement $"^{\prime \prime}$. Boudin proposait d'envoyer des commissaires dans les départements concernés. "Cette proposition a fait sourire et murmurer quelques membres de la Convention. Mais quoi Les représentants du Peuple ne doivent-ils donc paraître dans les départements que pour y présider des réquisitions, des destitutions, des incarcérations Quatre à cinq commissaires suffiront $»^{15}$. Les projets d'Eschasseriaux et de Rougier-Labergerie ne furent pas retenus. Et bien qu'il fût présenté au nom du Comité des subsistances et approvisionnements, du Comité de salut public et du Comité d'agriculture, celui de Boudin ne le fut pas davantage.

4 L'Assemblée préféra le rapport présenté le 3 frimaire (23 novembre) par Bourdon, député de l'Oise, quinze jours seulement après celui de Boudin. Celui-ci s'inspirait fortement du texte précédent. La grande différence, c'est que Bourdon étendait les mesures à l'ensemble de la République. Il ne reprenait pas à son compte, en revanche, l'idée des commissaires. Soutenu par le Comité d'agriculture, le texte de Bourdon fut voté à l'unanimité. Il avançait que l'adoption de son projet aurait, pour les subsistances de la Nation, les conséquences les plus heureuses. Les étangs, disait-il, couvrent en France 500000 arpents (210000 hectares)'. "Sur les terrains engraissés par les sédiments que les eaux y ont apportés depuis tant d'années, il suffit de répandre la semence sans préparation; et, pourvu qu'elle soit couverte par la charrue et la herse, on est presque certain d'une abondante moisson[...] À supposer [continuait Bourdon] qu'on ne puisse dessécher que 400000 arpents d'étangs, et en ne comptant que leurs produits qu'à six grains pour un, la masse des grains propres à la nourriture de l'homme serait toujours, pour l'année prochaine, augmentée de 2400000 setiers, c'està-dire que la subsistance de près d'un million de nos concitoyens se trouverait assurée par cette addition faite à l'agriculture ». De tels arguments emportèrent l'adhésion de la Convention. 
5 Le texte présenté était court: il ne comprenait que sept articles. Les deux premiers indiquaient l'urgence d'arriver promptement à des résultats. L'article premier décrétait que « tous les étangs seront mis à sec avant le 15pluviôse prochain par l'enlèvement des bondes et coupures de chaussées ». On accordait donc un délai de trois mois pour mettre à sec l'ensemble des étangs de la République! C'était peu. L'article premier révélait encore l'influence des sans-culottes et leur volonté de saper les opposants. Il visait les propriétaires et non les exploitants des étangs : si les étangs n'étaient pas vidés dans les délais impartis, les propriétaires seuls seraient tenus responsables des retards d'exécution: «Les étangs ne pourront être remis en eau, le tout sous peine de confiscation, au profit des citoyens non-propriétaires des communes où sont situés les étangs ». Compte tenu de la répartition de la propriété des étangs, la mesure touchait essentiellement la noblesse". Seuls pouvaient être maintenus en eau les étangs d'un arpent, nécessaires pour l'abreuvement des bestiaux. Comme le prévoyait l'article5, " ne sont pas considérés comme étangs, les réservoirs d'eau destinés à l'irrigation des prairies et à abreuver les bestiaux, pourvu qu'ils ne contiennent pas plus d'un arpent et s'ils ont une plus grande étendue, ils seront réduits à un arpent ». L'article2 stipulait que « le sol des étangs sera ensemencé en grain de mars ou planté en légumes propres à la subsistance de l'homme». Cette mesure répondait à la pression populaire qui souhaitait interdire la mise en herbe ou en légumineuse des terres jusque-là emblavées et augmenter la production des céréales pour nourrir les citadins et les soldats. Cet article était très prégnant puisqu'il violait le principe de la liberté des cultures. Mais il rejoignait le calcul économique des exploitants, ce que constatait Varenne de Fénille dès l'été 1793 : «Dans les années 1792-1793, les propriétaires ou les fermiers ont été portés par leurs intérêts à ensemencer un grand nombre d'étangs, le poisson étant tombé de valeur, et les grains étaient arrivés graduellement à un prix excessif ». Boudin soulignait également très clairement la même tendance : « Depuis que le prix des grains et des bestiaux va toujours croissant [...], le prix du poisson n'a pas suivi celui des autres denrées, on a été moins jaloux de conserver les étangs. On en a converti successivement en terres labourables et en prairies ». Le prix du marché foncier de la Brie confirmait ces affirmations. Les étangs de la commanderie de Bilbartaut par exemple, sur la commune de Jouarre, qui avaient été loués pour 4000 livres, plus 120 livres de rentes à l'abbesse de Jouarre en 1782, n'étaient plus évalués que pour un produit de 1600 livres en 1794. À l'inverse, le produit de la terre du domaine suivait un cours diamétralement opposé, signe du grand besoin de terre : évalué à $2500 l i v r e s$ en 1783, l'arpent de terre sur ce même domaine valait 3700 livres dixans plus tard". Les articles 4 et 7 du décret essayaient de combiner agriculture et meunerie « sont exceptés de dessèchement les étangs nécessaires pour alimenter les fossés des villes de guerre, les usines métallurgiques, le flottage, les papeteries, filatures, moulins à foulon et à scier pourvu que toutes ces usines aient été construites avant la présente loi »" et " il sera excepté de dessèchement ordonné à l'article1 les étangs indispensables pour le service des moulins et autres usines $»^{20}$.

6 Quatre jours après son adoption, le décret fut accompagné d'une instruction ${ }^{21}$. Celle-ci fut adressée aux administrations des districts et aux sociétés populaires ${ }^{2}$. Ce texte remettait bien dans son contexte la préoccupation du moment «La loi bienfaisante contribuera à détruire un des grands projets de nos ennemis, celui de nous affamer ». Il y était rappelé que la loi interdisait formellement la culture autre que celle dont l'homme se nourrissait. Le comité rappelait que le décret avait pour objet de « rendre à 
la santé et à la vie des milliers de Français qui moissonnent, chaque année, avant le terme marqué par la nature des maladies produites par les funestes émanations des étangs ». Le texte précisait encore que "la loi n'a voulu que des opérations non seulement très possibles mais aussi faciles et peu dispendieuses ». Ainsi, à partir du moment où l'eau des étangs pouvait s'évacuer par la levée des bondes ${ }^{23}$ ou la coupure des chaussées", il était évident que les pièces d'eau devaient être asséchées. Le décret précisait toutefois que les dessèchements ne devaient pas se faire au détriment des chaussées. Le législateur de l'an II avait trop le souci de conserver ces moyens de communication qui assuraient les échanges entre villages ${ }^{25}$. Enfin, il était recommandé de commencer le dessèchement des étangs inférieurs et, de proche en proche, de mener les travaux aux étangs les plus élevés ${ }^{26}$ : la vision systémique du réseau hydrologique jouait donc ici complètement. La question du dessèchement des étangs obligeait en effet à penser le réseau hydrologique dans sa globalitée souligné dans son exposé introductif « qu'on n'obtiendra aucun avantage si l'opération de dessèchement des étangs n'est pas universelle et prompte. Un seul étang laissé en arrière pourrait compromettre les productions de tous les autres $»^{23}$. Des difficultés dans l'écoulement des eaux entraînèrent le vote d'une autre loi un mois plus tard, le 8 ventôse an II (26février 1794). Son objet: l'écoulement des eaux dans une propriété voisine. On abordait là un problème qui allait être au centre de tous les débats juridiques du XIX ${ }^{\mathrm{e}}$ siècle concernant l'eau. Il s'agissait ici d'obliger les propriétaires à engager des frais pour l'écoulement de l'eau des étangs: "La loi concernant le dessèchement des étangs nécessite, pour le libre cours des eaux, le creusement de nouveaux fossés, le redressement ou l'augmentation des anciens fossés. Dans tous les cas, les propriétaires auxquels on a ordonné de dessécher leurs étangs doivent-ils indemnité aux propriétaires sur le terrain desquels les eaux prennent leurs cours? Point de doute, si les propriétaires des étangs en avaient entrepris le dessèchement de leur propre mouvement et pour leur plus grand intérêt. Mais il arrivera souvent que ces travaux utiles à la chose publique coûteront beaucoup plus aux propriétaires qu'ils ne leur produiront ainsi, il paraît que chaque Républicain doit faire pour l'intérêt général quelque sacrifice la décision sur cet objet est instante pour la prompte et entière exécution de la loi $»^{29}$.

7 La politique de dessèchement définie par les Jacobins fut conduite au niveau central par la Commission des subsistances puis par la Commission d'agriculture et des arts. Un conseil d'agriculture dépendant de la commission fut créé. Sur son avis, le Comité de salut public décida, le 2germinal an II (22 mars 1794), de souscrire à La Feuille du Cultivateur pour 2000 exemplaires. Cet organe de presse tint alors un rôle important : il fut chargé de vulgariser les principes de l'agriculture nouvelle et notamment les mesures concernant le dessèchement des étangs en application du décret du 14 frimaire ${ }^{\circ}$. Les numéros du journal devaient, à cette fin, être distribués par le soin de la commission dans les départements, les districts et les sociétés populaires.

8 Au niveau local, « les administrateurs du district sont chargés des dessèchements " ${ }^{n}$. Ils étaient aussi « tenus de demander aux municipalités et de faire passer à la Commission des subsistances les états de semence en légumes et en grains de mars qui leur manquerait pour les mettre en valeur $~{ }^{\prime}$. Dans la même logique, l'arrêté d'application demandait aux municipalités de « faire parvenir aux districts, dans la huitaine, un état détaillé des étangs, lacs et réservoirs existants dans leurs communes; d'indiquer l'étendue desdits lacs en faisant des observations sur ceux qu'il était indispensable de 
laisser subsister soit pour l'irrigation des prairies ou abreuvage des bestiaux, soit pour le service des moulins ou autres usines ". Tout cela entraîna une correspondance et l'établissement de statistiques qui font aujourd'hui le régal de l'historien".

Que le décret du 14 frimaire ait eu des buts politiques est évident. À partir du moment où il s'agissait d'une loi votée, on l'imagina forcément en rupture avec les pratiques antérieures. Les contemporains le vécurent d'ailleurs ainsi. Mais cette politique s'inscrivait, sur le fond, dans une longue histoire. Certes, on passait de la volonté de l'assèchement des marais à celui des étangs. La différence n'était pas mince alors que les marais étaient pour la plupart des terrains communaux, les étangs appartenaient au clergé et à la noblesse, ils symbolisaient le privilège seigneurial" on ne s'en prenait pas ici ni aux mêmes biens ni aux mêmes propriétaires. Mais les hommes qui impulsèrent cette politique - le plus représentatif de ces membres pour les questions de dessèchement d'étangs était Rougier-Labergerie - étaient ceux qui, avant la Révolution, souhaitaient un renouveau de l'agriculture: les membres de la ci-devant Société d'agriculture de Paris, physiocrates pour la plupart qui se retrouvaient tous ou presque au sein du conseil d'agriculture mis en place par la Commission des subsistances". Dans ces temps troublés, ils reprenaient un thème qui leur était cher et qu'ils avaient eu l'occasion de développer avant la Révolution pour les marais depuis longtemps, ils souhaitaient la mise en culture de ces terres. Ils adaptaient simplement ce souhait au contexte du moment. Et ils saisirent probablement l'occasion du choc politique du 10 Août pour faire avancer ce projet.

II-Mise en œuvre difficile

10 Le législateur, en décidant l'assèchement de l'ensemble des étangs de la République, était-il sûr de son fait? En tout cas, il avait besoin de s'en persuader : " En asséchant les étangs, on peut être sûr qu'ils seront desséchés cet hiver et qu'ils seront au printemps en état de recevoir la semence en grains propre à la nourriture de l'homme ». Même si des esprits indépendants et courageux - qui le payèrent fort cher d'ailleurs déclaraient le contraire. Ainsi Varennes de Fenille: «Les paysans sont forcés d'ensemencer à nouveau ces étangs qui, dans les deux années précédentes, ont produit sans repos du seigle ou du froment puis du sarrasin pendant la première année, de l'avoine pendant la seconde, le terrain épuisé par cette triple récolte. Ce terrain auquel les propriétaires et les fermiers seront dans l'impossibilité de procurer de l'engrais ne produira que la plus chétive moisson ${ }^{16}$.

11 Qu'en fut-il pour la Seine-et-Marne Les sociétés populaires impulsèrent la politique d'assèchement au plan local. Se mit alors en place un double rouage, l'administratif et le politique. Ce dernier était chargé de contrôler le premier : alors que la Commission des subsistances écrivait aux districts le 18 frimaire an II pour leur indiquer les actions à entreprendre pour l'assèchement des étangs, la même instance adressait, en parallèle, une circulaire aux membres des sociétés populaires". Le contraste de la teneur des propos adressés aux uns et aux autres est frappant : la première circulaire avait surtout un caractère administratif alors que la seconde sollicitait avec fougue le concours des sociétés populaires. Elle remettait sous leur surveillance l'application du décret : « Les corps administratifs chargés de l'exécution de la loi (celle du 14 frimaire) sont parfois lents à agir; donnez-leur, frères et amis, l'énergie républicaine. La Convention a décrété que les étangs seraient desséchés et ensemencés au printemps. Cette ressource, surveillée par vous, sera immense ; que tous les membres des sociétés populaires, les vrais sans-culottes, y appliquent leur brûlant civisme, leur lumière, leurs bras, s'il le 
faut : qui d'entre eux refuserait de tracer un sillon pour nourrir la liberté ». Le souffle républicain fut certainement efficace puisque cinq jours seulement après l'adoption du décret, les réactions du terrain remontaient : la municipalité de Bassevelle demandait ainsi par exemple aux administrateurs du district de Meaux la conservation de son étang'.

Mais les instances locales, pourtant politiquement sûres, parurent vite d'une efficacité insuffisante aux yeux des Montagnards parisiens pour faire face à la tâche assignée et surtout pour respecter les délais fixés. Boudin n'avait pas été suivi en novembre 1793 lorsqu'il avait proposé d'envoyer des commissaires visiter les étangs de la Sologne, de la Bresse et de la Brenne. La Convention comprit vite cependant que le délai fixé pour l'ouverture des bondes des étangs ne serait pas respecté si elle ne recourait pas à des moyens exceptionnels. Aussi, dès le 26 nivôse an II (15janvier 1794), cinq semaines seulement après la publication du décret, la Commission des subsistances envoyait, sur décision du Comité de salut public, des commissaires pour surveiller « les malveillants et la conduite des administrateurs et officiers municipaux "'. La commission en désigna cinq. L'un était serrurier (Yvart), les quatre autres présentés comme cultivateurs (Rougier-Labergerie, Dubrac, Villeneuve et Boutier). On peut s'étonner de voir figurer Rougier-Labergerie parmi ces commissaires. La raison est simple cependant. Mis en danger par le régime de la Terreur, Carnot le mit alors à l'abri en lui confiant cette mission qui l'éloignait pour un temps de Paris'. Le commissaire chargé de surveiller l'application du décret en Seine-et-Marne était moins illustre. Il s'agissait de Boutier, cultivateur à Sarguemine". La commission de l'agriculture et des arts invita ces cinq représentants à adopter une attitude prudente sur le terrain. Elle leur demandait d'éviter toute espèce de conflit avec les autorités constituées et ne rien faire sans son aveu. La Commission demandait aussi s'il était judicieux de n'autoriser que les cultures céréalières dans les étangs desséchés et souhaitait que les commissaires lui apportassent des éléments sur ce point.

Les cinq commissaires furent payés pour accomplir leur mission. Ils la remplirent de janvier à juin 1794. Boutier reçut 155 livres le 5 ventôse an II (23février 1794). C'était peu au regard des frais engagés pour se déplacer et se loger. Le territoire couvert par chacun était important en effet celui de Boutier couvrait huit départements : la Seineet-Marne, l'Aube, la Côte-d'Or, la Saône-et-Loire, le Jura, les Vosges, la Meurthe et enfin la Moselle. C'était beaucoup. Étroitement encadrés par les membres de la Commission d'agriculture et des arts, ces commissaires étaient aussi surveillés par les Jacobins du cru. Ce qui permit à la Commission de faire un rapport sur chacun". De même, les visites qu'ils réalisaient dans chaque département faisaient l'objet d'un compte rendu, aussitôt envoyé à la commission. Les 9 et 26ventôse (27 février et 16 mars 1794), celle-ci accusait ainsi réception des renseignements que Boutier avait collectés sur les dessèchements des étangs du district de Melun. En floréal (avril/mai 1794), elle estimait que ses statistiques étaient incomplètes". Le 8 prairial (27 mai 1794), elle lui en demandait de nouvelles; le 8 messidor (26 juin 1794), la commission lui signifiait que les dessèchements devaient être terminés et que ceux qui n'étaient pas achevés ne pourraient l'être en cette saison à cause du danger de pollution que cela provoquerait. La commission rappelait Boutier à la même date". Pourtant, en thermidor an II (juillet/ août 1794), celui-ci, inquiet de décevoir les instances parisiennes au cas où les statistiques demandées n'auraient pas été complétées, écrivait encore aux administrateurs des districts". 

ventôse an II (22 février 1794), la commission des subsistances demandait que les districts désignassent des citoyens pour «faire la visite des étangs susceptibles de dessèchement et faire la déclaration de ce qui leur serait nécessaire en blé de mars et légumes pour les mettre en valeur $"^{\prime *}$. Les districts firent diligence et nommèrent, en conséquence, des arpenteurs pour aller inspecter les communes. Dès février, ils furent chargés de procéder au recensement des étangs ". Les administrateurs du district de Meaux se distinguèrent par leur rapidité puisque Boutier reçut les renseignements dès le 8 ventôse ( 26 février) et les transmit à Paris le 13 (3 mars) ". Les autres districts mirent, en revanche, beaucoup moins de zèle à répondre. De leur côté, les ingénieurs des Ponts et Chaussées ne restaient pas inactifs, malgré les difficultés rencontrées pour l'accomplissement de leur mission" l'administration centrale leur demandait en brumaire « un état estimatif des frais que pourra occasionner le dessèchement des étangs " ; ils établissaient un rapport, en ventôse an II (février-mars 1794), pour juger de l'opportunité de conserver un étang sur la commune de Neufmoustier". Leurs actions furent limitées cependant sans grands moyens, l'administration des Ponts et Chaussées ne put envoyer dans tout le département des arpenteurs pour recenser les étangs à cause de la pénurie de géomètres ${ }^{32}$; les administrateurs des districts cherchèrent donc des gens moins qualifiés mais qui pouvaient remplir ces fonctions. Les administrateurs du district de Nemours nommèrent ainsi le 6 ventôse an II (24 février 1794) "un citoyen pour faire le recensement". De même à Meaux: le 18 thermidor an II (5 août 1794), le conseil général du district de Meaux, considérant que les procès-verbaux présentés par les communes n'étaient pas satisfaisants, décidait, lui aussi, de nommer deux géomètres pour remplir les états". l'opinion publique n'était pas favorable à ces dispositions. Toutefois, les manifestations de révolte furent rarissimes en Seine-et-Marne. Une seule opposition déclarée a été retrouvée dans les archives : en 1794, la commune de Courtry signalait que le citoyen Mérigot "ci-devant marquis de La Fère " s'opposait ouvertement à l'assèchement de son étang et à la pêche que les administrateurs des communes de Courtry et de Chelles avaient décidé de réaliser. L'absence d'oppositions signifiait-elle consentement? Certainement pas. L'explication de cette apathie générale fut donnée par le député Creuzé-Latouche" en mai 1795 au moment où celui-ci proposait l'annulation du décret du 14 frimaire : «L'opinion faisait de la moindre négligence, du moindre retard un sujet redoutable de dénonciation. Les cultivateurs et surtout les propriétaires entourés de délateurs, étaient accusés, menacés [...] Aussi, les intérêts des particuliers et des propriétaires n'ont presque point opposé de résistance $»^{s s}$.

16 Si les oppositions ouvertes furent rares, en revanche, les protestations résultant de l'application du décret du 14 frimaire furent très nombreuses". Elles arrivèrent rapidement. Les communes du district de Meaux formulèrent les premières au chef-lieu dès le 18 pluviôse an II (6février 1794), deux mois après le vote du décret ${ }^{\text {. }}$. Elles s'étalèrent jusqu'en septembre 1794. Certaines émanaient de possédants ayant récemment acquis un étang. La bonne affaire que certains croyaient faire en profitant de la mise sur le marché des étangs des religieux et des émigrés se révélait être un marché de dupes. Un certain Charles Boyer se plaignait ainsi aux administrateurs du district de Meaux, le 3 pluviôse an II (22 janvier 1794) : «J'ai acheté, il y a trente mois, 
33 arpents d'étangs pour prix et somme de 3400 francs. Je vous fais observer que je n'ai pas porté cet objet à un prix aussi exorbitant que par la possibilité d'établir un moulin à la chute des eaux desdits étangs. La loi sur le dessèchement détruit mes calculs et mes espérances ${ }^{\text {ti }}$. Mais ce genre de protestation fut rare. La plupart émanaient, non des propriétaires, trop menacés pour pouvoir eux-mêmes contrer les arguments des pouvoirs publics, mais des communes. Protestations que les districts transmettaient fidèlement sans chercher à les minimiser. Ils protestaient contre une application du décret qui nuisait aux intérêts des propriétaires et des fermiers mais aussi qui bousculait la pratique des usages. Les autres arguments étaient d'une nature différente. Ils peuvent être regroupés autour de sept thèmes :

17 -La nécessité de conserver les étangs afin de pouvoir faire tourner les moulins. Argument imparable, semble-t-il, à un moment où le pouvoir était obsédé par les problèmes d'approvisionnement de Paris en farine. Ainsi, les administrateurs du district de Meaux demandaient de conserver l'étang de Rougemont à Saint-Pathus riche de 7 à 8 moulins ${ }^{a}$. Boutier, le commissaire, notait, quant à lui, le 8 ventôse an II (26 février 1794), pour d'autres lieux que "dans l'étendue du district de Melun, il a été répondu que les étangs sont utiles pour les moulins à blé, que deux seulement étaient dans le cas du dessèchement : un à Echouboulain et un autre à Vaux-le-Pénil »"

18 -La nécessité de conserver les étangs pour l'abreuvement des bestiaux. Plusieurs communes du district de Meaux formulaient une telle demande en germinal an II (mars-avril 1794).

19 -La rupture des digues nuirait aux communications et aux échanges entre villages. Pour les étangs des cinq communes du district de Meaux, les statistiques révèlent que $70 \%$ de ces chaussées étaient déclarées utiles ou très utiles.

20 -Une commune s'étonnait qu'on puisse prendre un tel décret en avançant des raisons d'hygiène publique: le conseil général de Signy-Signet affirmait que son étang de Pereuse de 40 arpents (17 hectares) "n'a jamais produit aucun mauvais air contagieux" ". Une autre commune ajoutait même qu'en cas de dessèchement "la putréfaction de l'eau deviendrait funeste à la salubrité de l'air ".s.

21 -D'autres communes avançaient l'argument social. La commune de Croissy affirmait ainsi que son étang était rempli de roseaux qui servaient à couvrir les maisons des pauvres".

22 -Les moyens de parer à un risque d'incendie étaient aussi évoqués : «le défaut d'eau ôterait les moyens d'éteindre les incendies ${ }^{\prime \prime}$.

23 -Les conséquences des orages étaient encore avancées: la commune de Bassevelle soulignait ainsi que la quantité d'eau qui coulait des étangs de Lille, notamment pendant les orages de l'été, «perdrait les grains des autres étangs inférieurs qui sont aujourd'hui en culture $»^{*}$.

24 Plus redoutable que les oppositions et les protestations, l'inertie demeurait l'arme la plus efficace pour contrer les directives administratives. Cette inertie, les paysans la maniaient de main de maître. Ainsi, les géomètres-arpenteurs employés par les administrateurs du district de Meaux en août 1794 ne trouvaient aucun interlocuteur sur le terrain $\left({ }^{\circ}\right)$ :

"j'ai été dans plusieurs communes mais je ne trouvais personne à cause de la moisson. Les propriétaires trouvent toujours des inconvénients par crainte de la dépense $»^{70}$. 
III - Des effets pervers statistiques permettent de s'en rendre compte. Elles indiquent clairement pour la Seine-et-Marne l'entrée du processus d'assèchement dans le calcul économique des acquéreurs. Dans le district de Meaux, Boutier adressa le 13 ventôse an II (3 mars 1794) un état aux instances parisiennes : il permet d'avoir une vue précise de l'application du décret trois mois après sa promulgation, délai fixé d'ailleurs à l'article 1 . Sur les 1541arpents (647 hectares) répartis en 88 étangs, il recensait 1294 arpents (543 hectares) mis à sec: $84 \%{ }^{n}$. Dans le district de Provins, sur 32 étangs, 14 étaient asséchés à la même date ${ }^{n}$. À titre comparatif, pour les huit départements inspectés par Boutier, sur les 14490 arpents répartis sur 1536 étangs, 70 \% étaient asséchés à cette date. Les statistiques de Boutier permettent aussi de constater, pour le district de Meaux, un effort très différent d'assèchement selon les propriétaires. Les étangs appartenant à des Parisiens furent davantage asséchés que les autres : avec $82 \%$ des surfaces, ils devançaient les étangs possédés par les fermiers de 7 points et ceux de la République de 12. On peut supposer que les Parisiens, bien au fait des fluctuations du marché, cherchèrent plus que d'autres à profiter des cours des céréales".

L'application du décret du 14 frimaire amena plus d'ennuis que d'avantages. Ce que constatait le commissaire L'Héritier, membre de la Commission d'agriculture le 5 nivôse an III (25 décembre 1794)" « Dans trente-quatre départements, l'exécution de la loi n'a porté atteinte qu'à diverses ressources locales que les étangs procuraient à des fermes, hameaux et à des communes "15. Dans douze autres, le sort de l'agriculture, le service des ruisseaux et rivières pour la navigation, les usines et les flottages, semblent dépendre de l'existence d'un grand nombre d'étangs desséchés d'après la loi »".

L'Héritier ne classait pas le département de Seine-et-Marne dans la seconde catégorie". Malgré tout, la production agricole briarde n'eut rien à gagner dans l'assèchement de ses étangs. On a vu que l'article2 du texte prescrivait que seules les cultures utiles directement à l'homme pouvaient être autorisées dans les étangs desséchés. Or que constate-t-on sur le plateau briard ? Sur les 1294 arpents ( 660 hectares) mis à sec dans le district de Meaux, 599 arpents (306 hectares) furent convertis en pâturage (46\%), 608 arpents (310 hectares) en blé, chanvre, orge, colza ( $47 \%$ ), 76 arpents en foin et avoine $(6 \%), 8$ arpents en plantation $(0,6 \%)$ " . On remarque donc que plus de la moitié des terres asséchées furent mises en culture selon des principes contraires aux prescriptions législatives".

L'assèchement des étangs diminua bien évidemment la production piscicole. Le décret du 14 frimaire an II mit tout à coup sur le marché, dans les premiers mois de l'année 1794, une quantité importante de poissons. Varenne de Fénille s'en inquiétait : «C'est pendant l'automne qu'on empoissonne les étangs. Le tiers des étangs vont être empoissonnés. Le poisson ne grossit point pendant l'hiver ; en vidant tous les étangs indistinctement dans un mois, tout cet empoissonnement est anéanti. Quelle perte! Et croyez-vous citoyens députés que ce soit un moyen d'augmenter les subsistances? Il apparaîtra aussi une surabondance de poissons suivie d'une disette la plus absolue ». On aurait pu certainement saler ou mariner ces poissons mais le poisson d'eau douce, consommé par la classe aisée, était toujours vendu frais. En janvier 1794, Charles Boyer, le malheureux spéculateur ayant acheté un étang en Brie, déplorait lui aussi le gâchis

Annales historiques de la Révolution française, 325 | juillet-septembre 2001 
prévisible : « Dans un temps où l'on craint la rareté de la viande, la destruction absolue du poisson sera une ressource de moins pour la société »".

Les inconvénients résultant de l'assèchement des étangs ne se limitaient pas aux seuls domaines agricole ou piscicole. Ils touchaient aussi les moyens de communication. Les chaussées des étangs constituaient bien souvent des voies de communication essentielles entre villages. En rompant ces chaussées, on coupait bien souvent le chemin qui passait là. C'est la raison pour laquelle, reconnaissant l'existence de nombreux abus dans la façon dont les dessèchements étaient effectués, la Convention décida, le 21 pluviôse an II, qu'on ne couperait une chaussée d'étang que si toute autre solution était impossible. La Convention assouplissait ainsi un peu sa position. Elle donnait un prétexte à l'inapplication du décret. Mais n'était-ce pas déjà trop tard ? Le 1er fructidor an II (18 août 1794) Boutier estimait que les deux tiers des chaussées étaient déclarés utiles ou très utiles dans le district de Meaux ${ }^{\mathrm{at}}$. Or en thermidor an II (juillet-août 1794), $11 \%$ des chaussées des communes de Jouarre, Saint-Fiacre et Pierrelevée avaient déjà été rompues et $10,5 \%$ susceptibles de l'être dans un proche avenir.

IV - Une chaîne décisionnelle aveugle, terrifiante et terrifiée

De si piètres résultats questionnent sur la conduite de la politique agricole menée au niveau national. Comment les hommes au pouvoir ont-ils pu prendre des décisions qui semblent aberrantes aujourd'hui Essayer de répondre à cette question conduit à porter un regard sur le pilotage et le suivi des affaires agricoles durant la période montagnarde.

31 Le décret fut pris dans l'urgence et pour des raisons politiques une analyse rationnelle les en aurait certainement dissuadés. La précipitation fut mauvaise conseillère. Certes, il y avait urgence : il fallait armer la Nation et trouver des moyens de subsistance pour les armées. Toutes les ressources matérielles furent soumises à réquisition. Toutes les entreprises furent mises au service de la Nation : les forêts et les mines, les carrières, les papeteries... Il fallut aussi assurer l'approvisionnement de Paris. Quoi de plus tentant, dès lors, que de convertir les étangs situés aux portes de Paris, symboles détestés de l'Ancien Régime Assurément, la question des subsistances hantait les esprits des hommes durant cet automne 1793 et cet hiver 1794 : le 30ventôse an II (20 mars 1794), un prisonnier ayant habité La Nouvelle-Orléans nommé Olivier Prévost, propriétaire d'étangs, écrivait de la maison d'arrêt de Meaux pour proposer de cultiver $\mathrm{du}$ riz dans les terres humides desséchées de Seine-et-Marne ${ }^{\text {s. }}$. Cette anecdote illustre l'état d'esprit des gens de l'époque. Quinze mois plus tard, le député Creuzé-Latouche décrivit bien le contexte du moment : «L'opinion de la multitude, dans quelques cités, pensait que le dessèchement subit de presque tous les étangs allait faire affluer les subsistances $»^{\circ}$. Le peuple avait faim et les marais asséchés allaient produire ce qui manquait. En théorie du moins. En cela, les Physiocrates étaient aussi naïfs que les Montagnards. La nécessité de produire rapidement des céréales expliqua donc la précipitation donnée au cours des choses trois mois pour assécher les étangs, six pour produire les grains de mars. La Commission des subsistances ne semble pas avoir eu conscience des délais trop courts fixés pour l'application du décret puisque dès le 22nivôse (11janvier), dans la circulaire aux administrateurs des districts, elle demandait des renseignements sur le dessèchement des étangs «dont la culture ajouterait beaucoup à la richesse du territoire français ». 

en compétences que la France révolutionnaire n'avait pas. Pour faire face à l'effort de guerre, on avait, en août 1793, décrété la levée en masse. Creuzé-Latouche, critiquant la loi du 14 frimaire an II, en notait les conséquences ": "Il fallait des bras pour les travaux indispensables aux étangs desséchés. Il en fallait encore pour les mettre simplement en culture. Mais si ceux qui s'emploient aux cultures habituelles y suffisent à peine, comment pourrait-on en employer ailleurs? $\|^{\text {"5 }}$ L'argent manquait aussi tout autant que les bras. On imagine l'embarras des communes, propriétaires malgré elles des étangs des ci-devant nobles lorsque les lots n'avaient pas trouvé d'acquéreurs. À la pénurie de bras et d'argent, s'ajoutait la pénurie de semences de céréales. Conformément au décret du 14frimaire, les districts devaient demander à la Commission des subsistances les quantités de semences en légumes et grains de mars qui leur manquaient pour mettre en valeur les terres desséchées". Oui, mais les grains ne circulaient plus. Les hommes au pouvoir ont certainement minimisé ces moyens nécessaires à la conduite d'une politique d'assèchement.

Pour le suivi, les pouvoirs centraux étaient condamnés à piloter à l'aveugle. Les communes n'étaient pas encore familiarisées avec les nouveaux rouages administratifs. Les administrateurs du district de Melun faisaient le constat de ce laxisme administratif le 1er pluviôse an II (20 janvier 1794) concernant le suivi des dessèchements : « Sur les 116communes dont le district de Melun est composé, 39 seulement ont répondu. Nous allons prendre des mesures pour que nos opérations ne soient pas arrêtées par l'insouciance coupable des communes qui ne peuvent pas concevoir que pour mettre une administration à portée d'opérer régulièrement, il faut lui répondre négativement lorsqu'on n'a pas d'autre réponse à lui donner " ". La mise à l'écart des anciens notables, remplacés par des hommes pétris d'idées nouvelles mais bien souvent illettrés et peu enclins à remplir les colonnes des états statistiques envoyés par Paris n'arrangeait rien. Boutier, le commissaire, notait, en février 1794, que les districts de Provins et de Nemours étaient en retard pour rendre les statistiques demandées". "Je prie les citoyens commissaires de vouloir bien me faire passer le plus tôt possible à Dijon les réponses à ces questions $\|^{*}$. Un peu plus tard, il demandait des renseignements complémentaires aux districts : à celui de Meaux le nombre des étangs; à celui de Rozay leur situation; au district de Melun, la contenance des étangs nécessaire au fonctionnement des moulins. Dès le 27 pluviôse an II (15 février 1794), la Commission des subsistances admonestait les districts : «Quoi! La loi parle et vous ne lui avez pas obéi ? Citoyen, il nous en coûterait trop de vous croire coupables. Nous aimons mieux à penser que des obstacles vous ont arrêtés $"^{\circ}$. Le 2thermidor an II (20 juillet 1794), Boutier, à son tour, s'énervait et écrivait aux administrateurs des districts : «Voici la troisième circulaire que je vous adresse. N'auriez-vous pas reçu mes lettres ou vos réponses ne me sont-elles point parvenues ? Précision, célérité, salut et fraternité »". Il demandait au district de Meaux de lui envoyer réponse à Nancy, poste restante". Il écrivait, le jour même, aux administrateurs des districts: «Vous m'avez promis de m'adresser l'état récapitulatif à l'exécution de la loi du 14frimaire concernant le dessèchement des étangs [...] mais cela ne suffit pas : je dois fournir sous peu de jours l'état général à colonnes, contenant en tête le nom du département et du district; en marge, 1-le nom des communes et étangs, 2-la quantité de terrains couverts d'eau avant la loi, 3-la quantité de terrains desséchés, 4-la nature de leur production, grains ou pâturage, 5-la contenance par arpent d'ordonnance des terrains conservés en eaux, 
6-les motifs qui ont déterminé leur conservation $»^{\prime \prime}$. Ces réprimandes furent certainement inefficaces puisque le 13thermidor an II (31juillet 1794), Boutier n'avait toujours pas reçu les statistiques des districts de Melun, Rozay, Provins, Nemours et Nogent-sur-Seine". Seul le district de Meaux avait rempli sa copie dans les délais prescrits. Mais ces retards étaient-ils dus simplement à la négligence des administrateurs ? La cause de tels retards ne devait-elle pas aussi être recherchée dans la pénurie d'arpenteurs? Pour respecter la législation à la lettre, il en aurait fallu une multitude pour évaluer les travaux à entreprendre. Ce qui n'était pas le cas. La pénurie d'arpenteurs, déjà constatée sous l'Ancien Régime, était plus importante maintenant, compte tenu de l'ampleur et de la simultanéité des travaux demandés. Cela expliquait, en partie, les retards pris par les communes pour rendre les états statistiques dans les délais demandés".

manque de moyens, la sous-estimation des difficultés, la mauvaise lisibilité de l'application du décret n'expliquaient pas tout. L'aveuglement idéologique, le refus de considérer la nature telle qu'elle se présentait étaient aussi à mettre au compte des causes principales de l'échec de l'opération. Forts de leurs certitudes et contraints par la nécessité, les Montagnards poursuivirent l'application du décret du 14frimaire, envers et contre tous, malgré les observations de pur bon sens qui émanaient des provinces. Pourtant, dès le mois de janvier, des communes protestaient. Le commissaire Boutier émettait des doutes quant au respect du calendrier dès le 10 ventôse an II (28février 1794), trois semaines seulement après le délai prescrit: "Les étangs (de Rozay) ont été mis en pêche successivement et les eaux lâchées mais le dessèchement dans la plupart sera fort long; presque tous les fonds d'étangs ont beaucoup de vases ou de limon; les administrateurs du district s'adresseront à la Commission des subsistances pour savoir ce qu'il conviendra de faire à l'égard des terrains bourbeux dans lesquels les bêtes de trait ne pourront pénétrer $»^{*}$. Prudent, il rassurait cependant les membres de la commission de son loyalisme envers la politique fixée par Paris : «Je ne suis pas autorisé à faire remettre en eau sur les terrains profondément vaseux dont la culture sera impraticable pendant le printemps et les émanations dangereuses pendant l'été ; je me suis borné à consigner ces faits ; sans leur indiquer qu'elle serait leur règle de conduite ». Il concluait en suggérant d'adopter une règle de bon sens : « Il semble qu'à cet égard, on pourrait s'en rapporter aux connaissances locales des administrations ». Quatorze jours plus tard, Boutier s'enhardissait un peu plus et, le 24ventôse an II (14 mars 1794), il faisait part, à la Commission des subsistances et approvisionnements, de ses doutes sur l'application du décret. Un peu plus tard, il adressait les remarques des communes et il envoyait aux membres de la Commission des subsistances les «questions qui m'ont été posées par les administrateurs des départements de Seine-et-Marne et Aube :

1-Faut-il dessécher les étangs dont le sol est notoirement reconnu pour infertile par les essais faits antérieurement pour les cultiver?

2-La limitation pour les abreuvoirs à un arpent privera totalement d'eau le bétail des cultivateurs dans les temps de sécheresse. L'année dernière, une étendue de 20 arpents [ 8,5 hectares] couverts d'eau a été réduite à trois.

37 3-Il y a des étangs dont le sol ne sera propre qu'au vain pâturage ; il en est d'autres qui formeront des prés ; ces deux productions naturelles qui n'exigent ni soin ni dépenses paraissent préférables à une culture très difficile dans ce moment. 

des commissions à faire remonter des informations qui auraient pu déplaire. Les membres de la Commission des subsistances avaient bien conscience des difficultés : il leur suffisait de lire les rapports de Boutier et ceux de ses collègues; ils avaient d'ailleurs adressé, le 18 ventôse (8 mars 1794), un mémoire au Comité d'agriculture pour attirer son attention sur les incohérences du décret du 14 frimaire. On ignore la réponse mais on peut la deviner puisqu'une semaine plus tard, le 26ventôse an II (16 mars), le président de la commission avouait son impuissance : « La commission ne peut se dispenser de la loi, elle ne peut ni la modifier ni l'interpréter. Elle avait engagé le Comité d'agriculture à solliciter de la Convention quelques articles supplémentaires mais il paraît que l'on aurait plus d'inconvénients à modifier cette loi qu'à la laisser telle qu'elle existe $»^{*}$. Le même jour, le président de la Commission des subsistances invitait donc Boutier, «à poursuivre sa mission dans les départements avec zèle ". Le Comité d'agriculture se décida tout de même, un mois plus tard, le 29 floréal an II (18 mai 1794), à transmettre un rapport au Comité de salut public sur les dessèchements. Mais quel était le motif de ce texte? N'avait-il pas pour but de dédouaner les membres de la commission et de justifier les retards prévisibles? «Si on a toujours reconnu que le dessèchement était indispensable, on a été effrayé de la difficulté de l'exécution et l'on n'a pas été d'accord sur les moyens[...] Dès cette année, des milliers d'arpents rendus à la culture augmenteront sensiblement la masse de nos subsistances. Les marais sont plus considérables que les étangs et demandent plus de temps et de précautions pour être desséchés. Ils exigent plus de moyens dispendieux et moins simples $»^{100}$.

Conclusion remarque Octave Festy ${ }^{\text {ton }}$, combien de prés nécessaires à l'alimentation des bestiaux furent sauvés par la mauvaise volonté des cultivateurs à appliquer le décret du 14 frimaire an II ? Et combien de journées d'hommes et de chevaux furent économisées. Combien de semences destinées à faire produire quand même des terrains fangeux ou autrement stériles furent aussi épargnées?

41 Certes, mais au-delà de l'histoire événementielle, il convient aussi de resituer ce décret dans un plus long terme. Comme sous l'Ancien Régime, les assèchements furent encouragés, au nom de l'efficacité économique, au nom des principes d'hygiène. L'objet se déplaça simplement des marais aux étangs. La finalité politique était toute autre mais les mêmes arguments présidaient à l'entreprise. La permanence et l'influence des membres de la ci-devant Société d'agriculture de Paris et notamment RougierLabergerie et d'autres encore étaient pour beaucoup dans cette situation. Ils reprenaient à leur compte une politique menée depuis plusieurs décennies avec plus ou moins de bonheur par les différentes administrations de l'Ancien Régime. Ils les adaptèrent simplement aux événements politiques du moment.

Ces assèchements précipitent aussi une adaptation. Ils marquent le coup de grâce d'une production piscicole déjà sur le déclin depuis le milieu du $\mathrm{xVIII}^{\mathrm{e}}$ siècle. Les grandes enquêtes agricoles de l'Empire manquent cruellement pour la Seine-et-Marne il est donc très difficile de savoir si certains propriétaires remirent en eau leur étang sous le 
Directoire, le Consulat ou l'Empire. Ce qui est sûr cependant, c'est qu'au moment de l'établissement du cadastre réalisé pour cette région en 1831, tous les étangs, à l'exception d'un, avaient disparu.

\section{NOTES}

1.Pour suivre cette réactivité sur toute la période, voir Jean-Michel Derex, Intérêts privés, intérêts généraux et intérêts communautaires : la gestion de l'eau en Brie (fin de l'Ancien Régime-fin du XIXe siècle), Université Paris IV-Sorbonne, doctorat d'histoire, 1999 ; La gestion de l'eau en Brie de la fin de l'Ancien Régime à la fin du XIXe siècle, Paris, l'Harmattan, 2001.

2.Octave FESTY a étudié le décret du 14 frimaire. Voir O. FESTY, Les conditions de production et de récolte des céréales. Étude d'histoire économique :1789-1795, Paris, Gallimard, 1947, pp.174-189. À notre connaissance, l'application de ce décret n'a pas, en revanche, fait l'objet d'études.

3.Le dessèchement des étangs peut être suivi très précisément. Les cartons des Archives nationales (cartons F10 209A, 261, 309, 310, 312, 313, 315, 331 et 322) et ceux des archives départementales de Seine-et-Marne (cartons L 520,521) donnent de nombreux renseignements.

4.ESCHASSERIAUX (aîné) Joseph (1753-1823) avocat, élu député de la Charente-Inférieure à l'Assemblée législative en 1791, puis à la Convention en 1792. Vota la mort du Roi et siégea à la Montagne. Élu au Conseil des Cinq-Cents en 1795.

5.ROUGIER-LABERGERIE ou ROUGIER de la BERGERIE (1757-1836) : riche propriétaire terrien, il s'adonne à l'agriculture. Il proposa au Roi, en 1788, un Mémoire sur les abus qui s'opposent aux progrès de l'agriculture que la Société d'Agriculture de Paris reprit en 1789. Membre de la municipalité de Paris en 1789. Élu député de l'Yonne en 1791.

6.Boudin (1756-1797) agriculteur à Châteauroux. Élu député de l'Indre en 1792. Il prit des positions modérées mais resta toujours l'adversaire des prêtres dont il demanda l'arrestation et la déportation.

7.Arch. nat., AD IV22, octobre 1793. Rapport de Boudin, député de l'Indre à la Convention, Mémoire sur le dessèchement et la mise en culture des étangs de la Sologne, de la Bresse et de la Brenne.

8.Rougier-Labergerie faisait bien la distinction dans son rapport « Il ne faut pas confondre les étangs marécageux avec ceux dont les eaux limpides reposent sur un sol sablonneux et qui sont utiles ou nécessaires à des irrigations, à des usines, à la salubrité de l'air et à la fertilité des champs ».

9.Les Montagnards décrétèrent, le 3 juin, la vente des biens des émigrés par petits lots payables en dix ans. Cette décision avait des répercussions directes sur le sujet traité ici.

10.Décret sur le dessèchement des marais.

11.Arch. nat., AD IV22, octobre 1793. Rapport de Boudin, op. cit. 
12.Arch. nat., AD XVIIIc 310 et AD IV 22, septembre 1792. ESCHASSERIAUX, Rapport de projet de décret présenté à la Convention nationale au nom des Comités d'agriculture, des finances, de l'aliénation et des domaines sur le dessèchement des marais (non daté).

13.Jean BouTIER, Campagnes en émoi : révolte et Révolution en Bas-Limousin : 1789-1800, Treignac, Les Monédières, 1987, pp. 118-124.

14.Boudin, op. cit.

15.Ibid.

16.Soulignons le caractère fantaisiste de ces chiffres. Eschasseriaux avançait un chiffre trois fois supérieur, un an auparavant.

17. À cette date, les communautés ecclésiastiques n'étaient plus visées puisqu'elles étaient déjà dissoutes. Une approche régionale de cette répartition de la propriété des étangs peut être faite à partir de notre thèse sur la Brie J.-M. DEREX, op. cit.

18.Gombert-Alexandre RETHORE, La Commanderie de Bilbartaut et ses dépendances, Meaux, Le Blondel, 1887, p.71.

19.Décret du 14 frimaire an II, article 4.

20.Ibid, article 7.

21.Arch. nat., $\mathrm{F}^{10} 310$ et 254,9 nivôse an II.

22.Pierre CARON, La Commission des subsistances de l'an II, Procès-verbaux et actes, Paris, Ernest Leroux, fasc. I, pp.103-104.

23.Une bonde est la fermeture du trou d'écoulement des eaux d'un étang.

24.La chaussée est le remblai servant à retenir l'eau et à la diriger vers un point déterminé.

25.Les chaussées d'étangs servaient, en effet, bien souvent de voie de communication entre les villages ou les hameaux.

26. Arch. nat., $\mathrm{F}^{10}$ 310, brumaire an II.

27.Jusque-là, la vision systémique des cours d'eau n'avait pas été réellement abordée. À peine l'avait-on effleurée dans l'article 7 de la loi du 5 juin 1791 avec l'obligation de curage pour le libre écoulement des eaux. Mais on n'abordait alors que les cours d'eau non navigables mais flottables.

28.Les étangs, par leur disposition en batterie, demandaient une coordination d'ensemble si l'on voulait tous les assécher. Il n'était pas possible de mettre à sec un étang sans procéder de la même manière pour les autres, situés en amont ou en aval. 29.Arch. nat., $\mathrm{F}^{10} 310$, ventôse an II.

30.Octave FESTY, «L'Agriculture pendant la Révolution. Les journaux d'agriculture et le progrès agricole (1789-anVIII) », Revue d'histoire économique et sociale, 1950, p.35. Créé au début de l'année 1788, ce journal allait traverser toute la période révolutionnaire jusqu'en l'anVIII. Cet organe de presse, qui faisait paraître, deux fois par semaine, un numéro, était animé par J.-B.Dubois de Jancigny, avocat. Il s'adjoignit le concours de Broussonnet, docteur en médecine, membre de l'Académie des Sciences, secrétaire perpétuel de la Société royale d'agriculture. Heurtault de Lammerville, entre autres, collabora aussi à ce journal. Collaborèrent aussi des spécialistes des questions agricoles et des députés : Parmentier, Rougier-Labergerie, Creuzé-Latouche, Varenne de Fenille, Vilmorin, Coupé, Eschasseriaux. Si la Commission favorisa La Feuille du Cultivateur, le régime de la Terreur considérait comme suspects plusieurs agronomes qui y écrivaient : Dubois, Parmentier, Varenne de Fenille. Les auteurs du journal étaient opposés aux méthodes de la Terreur et le journal s'abstint de prendre parti dans les luttes politiques de l'époque. 
31.Décret du 14 frimaire an II, article 3.

32.Ibid, article 6.

33. Arch. nat., $\mathrm{F}^{10} 310$, décembre 1793. Instruction sur l'exécution du décret du 14frimaire relatif au dessèchement des étangs. L'administration centrale donnait des directives sur l'application des dessèchements à mener. On peut constater l'irréalisme de certaines de ces directives. Ainsi, concernant les miasmes et autres vapeurs « mortelles » émanant des étangs, les bureaux parisiens estimaient, par exemple, que le meilleur moyen de les éloigner était d'allumer des feux sur le bord des étangs pour que le vent ne les emportât pas sur les habitations voisines : «On préviendra au reste le mauvais effet de ces émanations par l'usage de l'ail et de boisson au vinaigre ».

34.Symbole seigneurial, les étangs étaient assortis de privilèges. Voir Geoffroy de GISLAIN, Étangs, garennes et colombiers, thèse de droit, Paris II, 1977.

35.Sous la Terreur, la Commission des subsistances se dota d'un conseil d'agriculture composé des membres de la ci-devant Société d'agriculture de Paris. Le régime de la Terreur considéra cependant comme suspects plusieurs de ses membres.

36.Arch. nat., $\mathrm{F}^{10} 321,1794$.

37.Arch. nat., $\mathrm{F}^{11} 268,18$ frimaire an II.

38.Arch. dép. Seine-et-Marne, L 521, 19 frimaire an II.

39.Pierre CARON, op. cit., p.246.

40.Arch. nat., $\mathrm{F}^{10}$ 209A, 26 nivôse an II.

41.Arch. nat., $\mathrm{F}^{10}$ 261, nivôse an II. En l'an II, Boutier vivait à Paris. Il assurait avoir cultivé avec succès des propriétés et desséché quelques étangs. Il était membre de la Société libre d'économie rurale et, à ce titre, il avait fait imprimer plusieurs mémoires. 42.Arch. nat., $\mathrm{F}^{10} 209 \mathrm{~A}$, floréal an II. Concernant Boutier, la commission estimait « qu'il offre beaucoup moins de résultats que les autres ".

43.Ibid.

44.On ignore si Boutier reçut à la fin de sa mission de nouveaux frais de mission pour solde de tout compte.

45.Arch. nat., $\mathrm{F}^{10} 310$, thermidor an II.

46.Ibid., 4 ventôse an II.

47.Arch. nat., $\mathrm{F}^{10}$ 321, ventôse an II.

48.Arch. nat., $\mathrm{F}^{10}$ 322, 3 ventôse an II. Des statistiques étaient encore demandées à de nouveaux commissaires-géomètres par les districts le 18 thermidor (arch. dép. de Seine-et-Marne, L 519) et ces enquêtes se poursuivaient toujours en fructidor (arch. de Seine-et-Marne, L 520, 18 fructidor an II).

49.Arch. dép. Seine-et-Marne, L43, 26 juin 1793.

50.Arch. nat., $\mathrm{F}^{14} 600$, brumaire an II.

51.Arch. nat., $\mathrm{F}^{10} 313$, ventôse an II.

52.Cette pénurie d'arpenteurs était aussi enregistrée dans le monde forestier.

53.Arch. nat., $\mathrm{F}^{10} 322,6$ ventôse an II.

54.Arch. dép. Seine-et-Marne, L519, 18 thermidor an II.

55.Arch. nat., $\mathrm{F}^{10}$ 312, et arch. dép. Seine-et-Marne, L 521, février 1794.

56.Arch. nat., $\mathrm{F}^{10}$ 31, 2 février 1794.

57.CREUZÉ-LATOUCHE Jacques-Antoine (1749-1800) avocat. S'intéresse à l'agronomie.

Membre de la Société royale d'agriculture de Paris. Membre du club des Jacobins en mars 1791, élu député de la Vienne à la Convention en 1792. Nombreux amis parmi les Girondins.

58.Arch. nat., AD IV 22, mai 1795. 
59.Un recensement départemental des protestations émises n'est pas envisageable, les données étant trop parcellaires.

60.Arch. dép. de Seine-et-Marne, L520, 18 pluviôse an II.

61.Arch. dép. Seine-et-Marne, L 521, 3 pluviôse an II.

62.Arch. nat., $\mathrm{F}^{10}$ 312, pluviôse an II.

63.Arch. nat., $\mathrm{F}^{10}$ 309, 8 ventôse an II.

64.Arch. dép. Seine-et-Marne, L 521, pluviôse an II.

65.Arch. nat., $\mathrm{F}^{10}$ 313, pluviôse an II.

66.Arch. dép. Seine-et-Marne, L521, pluviôse an II.

67.Arch. nat., $\mathrm{F}^{10}$ 313, pluviôse an II.

68.Arch. dép. Seine-et-Marne, L521, pluviôse an II.

69.Vieille habitude paysanne déjà rencontrée au XVIIesiècle lors de la constitution des réserves forestières.

70.Arch. dép. Seine-et-Marne, L 520, août 1794.

71.Arch. nat., $\mathrm{F}^{10}$ 309, ventôse an II.

72.Le district de Provins n'a pas fourni les surfaces.

73.Les étangs briards offrirent un refuge particulièrement apprécié au papier-monnaie des créanciers privilégiés de l'État, les fournisseurs. Ainsi, le principal acquéreur des étangs de la région de Jouarre était un certain Bayard, fournisseur de viande aux Invalides.

74.Arch. nat., $\mathrm{F}^{10}$ 310. Rapport général sur les étangs, fait au Comité d'agriculture et des arts par la Commission d'agriculture et des arts, 5 nivôse an III, signé par les commissaires Berthollet, L'Héritier et Tissot, adjoint par intérim.

75.Les départements étaient les suivants : Isère, Bouches-du-Rhône, Gard, Marne, Saône-et-Loire, Vosges, Creuse, Haute-Vienne, Jura, Eure-et-Loir, Sarthe, Orne, Mayenne, Loire-Inférieure, Seine-et-Marne, Meurthe, Haute-Saône.

76.Les départements étaient les suivants : Ain, Rhône, Loire, Nièvre, Cher, Loir-et-Cher, Loiret, Yonne, Indre, Aube, Dordogne, Côte d'Or, Oise.

77.Arch. nat., AD XVIIIc/310, 5 nivôse an III.

78.La production de foin donna, en moyenne, 40 quintaux à l'hectare en l'an II et 12 quintaux à l'hectare pour l'avoine. Les statistiques ne donnent pas le rendement des terres converties pour la culture du blé, ce qui est surprenant, compte tenu des nécessités du moment. Signalons toutefois quelques rares terres exceptionnelles avec 588 quintaux de foin à l'hectare!

79.Arch. nat., $\mathrm{F}^{10} 309$, thermidor an II.

80.Arch. dép. Seine-et-Marne, L 521, janvier 1794.

81.Arch. nat., $\mathrm{F}^{10} 315$, fructidor an II.

82.Arch. nat., $\mathrm{F}^{10} 331,30$ ventôse an II.

83.Arch. nat., AD IV22. CREUZÉ-LATOUCHE, Rapport fait au nom du Comité d'agriculture et des arts sur la loi du 14 frimaire an II, en mai 1795.

84.Dans le domaine sylvicole, on constata également un arrêt des défrichements durant la période révolutionnaire.

85.Arch. nat., AD IV22, mai 1795.

86.Décret du 14 frimaire, article 6.

87.Arch. nat., $\mathrm{F}^{10}$ 322, 1er pluviôse an II.

88.Ibid., février 1794 .

89.Arch. nat., $\mathrm{F}^{10}$ 309, février 1794.

90.Pierre CARON, op. cit., p. 358, 27 pluviôse an II. 
91.Arch. nat, $\mathrm{F}^{10} 310,2$ thermidor an II.

92.Arch. dép. Seine-et-Marne, L 521, thermidor an II.

93.Arch. nat., $\mathrm{F}^{10} 310$, thermidor an II.

94.Ibid.

95.Arch. nat., $\mathrm{F}^{10} 310,1794$.

96.Arch. nat., $\mathrm{F}^{10}$ 309, 10 ventôse an II.

97.Arch. nat., $\mathrm{F}^{10} 310,24$ ventôse an II.

98.Arch. nat., $\mathrm{F}^{10}$ 309, 26 ventôse an II.

99.Arch. nat., $\mathrm{F}^{10} 209 \mathrm{~A}, 29$ floréal an II.

100.Arch. nat., $\mathrm{F}^{10}$ 310, 26 ventôse an II.

101. Octave FESTY, Les conditions de production..., op. cit., 1947, p. 430.

\section{RÉSUMÉS}

Le décret du 14 frimaire an II décidait l'assèchement de tous les étangs de la République. Voté pour des raisons de circonstances autant qu'idéologiques, il faut aussi replacer cette décision dans la politique d'assèchement menée depuis le milieu du XVIII ${ }^{\mathrm{e}}$ siècle. Menée d'une manière brutale et précipitée, l'application du décret suscita de nombreuses oppositions dans le pays. Elle révéla la mauvaise appréciation de la réalité hydrologique des milieux politiques parisiens.

The Decree of 14 Frimaire Year II on the Drainage of Ponds : Wild Hopes and Dismal Results. The Implementation of the Decree in Brie. The decree of 14 Frimaire YearII prescribed the drainage of ponds throughout the Republic. Adopted for circumstantial as much as ideological reasons, the decision needs also to be viewed in the context of the drainage policy pursued since the middle of the eighteenth century. Brutally and hastily conducted, the implementation of the decree gave rise to widespread opposition in the country. It revealed the poor assessment made of hydrological reality by political circles in Paris.

\section{INDEX}

Mots-clés : Brie, dessèchement, législation révolutionnaire, étangs

\section{AUTEUR}

JEAN-MICHEL DEREX

Docteur en histoire 